\title{
Acylierung von Aromaten mit Carbonsäure-dihalogenphosphorsäure-anhydriden ${ }^{1)}$
}

\author{
Franz Effenberger*, Gerd Konig ${ }^{2)}$ und Herbert Klenk \\ Institut für Organische Chemie der Universitát Stuttgart, \\ Pfaffenwaldring 55, D-7000 Stuttgant 80
}

Eingegangen am 4. Juli 1980

\begin{abstract}
Aryl- und Alkylcarbonsăure-dihalogenphosphorsăure-anhydride 1, 2 bzw. 7, 8 reagieren mit aktivierten Aromaten (Alkylbenzolen, Anisol) auch ohne Zugabe von Friedel-Crafts-Katalysatoren in guten Ausbeuten zu Arylketonen 4, 9. Die gemischten Anhydride 7, 8 konnen auch in situ durch Mischen der Carbonsäureanhydride mit den Dihalogenphosphorsăure-anhydriden hergestellt und umgesetzt werden, was insbesondere für die weniger stabilen gemischten Anhydride von Nutzen ist.
\end{abstract}

\section{Acylation of Benzenes with Carboxylic Dihalophosphoric Anhydrides"}

Aryl ketones 4, 9 are formed in good yields without the addition of Friedel-Crafts catalysts from activated arenes (e. g. alkylbenzenes, anisole) and aryl- and alkylcarboxylic dihalophosphoric anhydrides 1,2 , and 7,8 , respectively. The anhydrides 7,8 may be prepared in situ by mixing the respective carboxylic and dihalophosphoric anhydrides. This procedure is especially advantageous in care of the less stable compounds.

Wir haben vorstehend über die Darstellung gemischter Anhydride aus Carbonsauren und Dihalogenphosphorsäuren berichtet ${ }^{3)}$. Aufgrund der guten Austrittstendenz von Dihalogenphosphat-Ionen ") war zu erwarten, daß diese Verbindungen ein hohes Acylierungspotential besitzen und daß sie deshalb, ähnlich wie Carbonsäure-trifluormethansulfonsăure-anhydride ${ }^{5}$, in der Lage sein sollten, Aromaten auch ohne Verwendung von Friedel-Crafts-Katalysatoren zu acylieren.

\section{Acylierung mit Arylcarbonsäure-dihalogenphosphorsäure-anhydriden 1 bzw. 2}

Da die gemischten Anhydride mit aromatischen Carbonsäuren, im Gegensatz zu den Anhydriden mit aliphatischen Carbonsäuren, zum Teil in reiner Form zugänglich sind $^{3)}$, haben wir diese als isolierte Verbindungen mit einem Überschuß an Aromaten ohne oder mit Lösungsmittel bei verschiedenen Temperaturen zu Diarylketonen 4 umgesetzt ${ }^{()}$(Tab. 1). Bei den bislang nicht in Reinsubstanz isolierten Anhydriden $\mathbf{1 b}, \mathbf{d}$ und e wurden die durch Umsetzung der entsprechenden symmetrischen Carbonsäureanhydride und Dichlorophosphorsäure-anhydrid erhaltenen Rohprodukte eingesetzt .

Die in Tab. 1 zusammengefaßten Ergebnisse zeigen, daß die Acylierung von Aromaten mit den gemischten Anhydriden 1 bzw. 2 auch ohne Verwendung von FriedelCrafts-Katalysatoren möglich ist. Gute Ausbeuten an Ketonen werden dabei allerdings nur bei den reaktiveren Aromaten erreicht, wobei bei Raumtemperatur Anisol etwas und Thiophen sogar stark exotherm reagieren. 


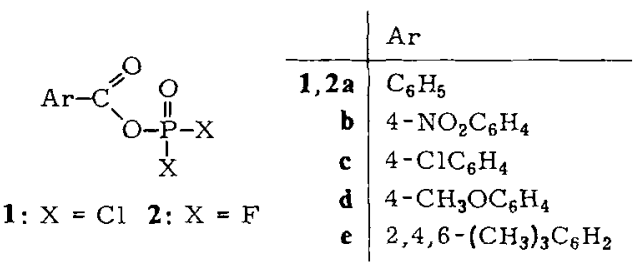

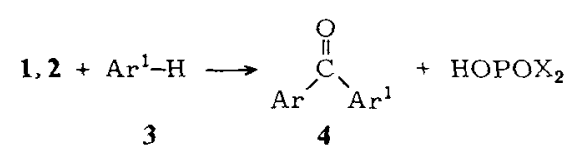

\begin{tabular}{|c|c|c|c|c|c|}
\hline & Ar & $A r^{2}$ & & Ar & $\mathrm{Ar}^{1}$ \\
\hline $3 a, 4 a$ & $\mathrm{C}_{6} \mathrm{H}_{5}$ & $\mathrm{C}_{6} \mathrm{H}_{5}$ & $4 j$ & $4-\mathrm{NO}_{2} \mathrm{C}_{6} \mathrm{H}_{4}$ & \\
\hline $3 b, 4 b$ & $\mathrm{C}_{6} \mathrm{H}_{5}$ & $4-\mathrm{ClC}_{6} \mathrm{H}_{4}$ & $4 k$ & $4-\mathrm{ClC}_{6} \mathrm{H}_{4}$ & $2,4,6-$ \\
\hline $3 c, 4 c$ & $\mathrm{C}_{6} \mathrm{H}_{5}$ & $2(4)-\mathrm{CH}_{3} \mathrm{C}_{6} \mathrm{H}_{4}$ & 41 & $4-\mathrm{CH}_{3} \mathrm{OC}_{6} \mathrm{H}_{4}$ & $\left(\mathrm{CH}_{3}\right)_{3} \mathrm{C}_{6} \mathrm{H}_{2}$ \\
\hline $3 d, 4 d$ & $\mathrm{C}_{6} \mathrm{H}_{5}$ & $2,5-\left(\mathrm{CH}_{3}\right)_{2} \mathrm{C}_{6} \mathrm{H}_{3}$ & $4 m$ & $2,4,6-\left(\mathrm{CH}_{3}\right)_{3} \mathrm{C}_{6} \mathrm{H}_{2}$ & \\
\hline $3 \mathrm{e}, 4 \mathrm{e}$ & $\mathrm{C}_{6} \mathrm{H}_{5}$ & $2(4)-\mathrm{CH}_{3} \mathrm{OC}_{6} \mathrm{H}_{4}$ & $4 n$ & $4-\mathrm{CH}_{3} \mathrm{OC}_{6} \mathrm{H}_{4}$ & $2,5-\left(\mathrm{CH}_{3}\right)_{2} \mathrm{C}_{6} \mathrm{H}_{3}$ \\
\hline $3 \mathrm{f}, 4 \mathrm{f}$ & $\mathrm{C}_{6} \mathrm{H}_{5}$ & $2,4,6-\left(\mathrm{CH}_{3}\right)_{3} \mathrm{C}_{6} \mathrm{H}_{2}$ & 40 & $4-\mathrm{ClC}_{6} \mathrm{H}_{4}$ & \\
\hline $3 \mathrm{~g}, 4 \mathrm{~g}$ & $\mathrm{C}_{6} \mathrm{H}_{5}$ & $2-\mathrm{C}_{4} \mathrm{H}_{3} \mathrm{~S}$ & $4 p$ & $\left.4-\mathrm{CH}_{3} \mathrm{OC}_{6} \mathrm{H}_{4}\right\}$ & $2(4)-\mathrm{CH}_{3} \mathrm{C}_{6} \mathrm{H}_{4}$ \\
\hline $4 h$ & $4-\mathrm{ClC}_{6} \mathrm{H}_{4}$ & $2(4)-\mathrm{CH}_{3} \mathrm{OC}_{6} \mathrm{H}_{4}$ & $4 q$ & $4-\mathrm{NO}_{2} \mathrm{C}_{6} \mathrm{H}_{4}$ & $\mathrm{C}_{6} \mathrm{H}_{5}$ \\
\hline $4 i$ & $4-\mathrm{CH}_{3} \mathrm{OC}_{6} \mathrm{H}_{4}$ & $4-\mathrm{CH}_{3} \mathrm{OC}_{6} \mathrm{H}_{4}$ & & & \\
\hline
\end{tabular}

Die Beschränkung der Anwendung dieser Acylierungsmethode auf aktivierte Aromaten hat seine Ursache in der Thermolabilität der gemischten Anhydride. Die Chlorderivate 1 zerfallen oberhalb $100^{\circ} \mathrm{C}$ ziemlich rasch unter Bildung von Carbonsäurechloriden, wohingegen die Fluorverbindungen 2 wesentlich stabiler sind, so daß auch über einen längeren Zeitraum bei erhöhter Temperatur umgesetzt werden kann und damit wesentlich bessere Ausbeuten an Ketonen erreicht werden (z. B. bei Toluol nach $16 \mathrm{~h}$ bei $115^{\circ} \mathrm{C} 62 \%$ Ausbeute). Unter vergleichbaren Reaktionsbedingungen ergeben die Umsetzungen von Anisol, Mesitylen und Thiophen mit 1a und 2 a vergleichbare KetonAusbeuten. Die relativ schlechte Ausbeute (41\%) bei der Acylierung von Mesitylen mit dem in situ hergestellten 4-Nitrobenzoesäure-dichlorophosphorsäure-anhydrid (1 b) ist nicht die Folge geringerer Reaktivität von 1 b im Vergleich zu 1a oder 1c-e, sondern die spektroskopisch beobachtbare, unvollständige Bildung von $\mathbf{1 b}$ aus den symmetrischen Anhydriden ${ }^{3)}$.

Abweichend von den normalen Friedel-Crafts-Acylierungen ist die Isomerenverteilung bei der Acylierung von Toluol. Während z. B. bei der Friedel-Crafts-Acylierung von Toluol ${ }^{7)}$ ca. $9 \%$ ortho-Isomeres entstehen, haben wir bei allen von uns durchgeführten Acylierungen von Toluol stets $20-22 \%$ ortho-Anteil erhalten (Tab. 1). Trotz der starken Begünstigung der ortho-Reaktion in diesen Fällen - was auf ein sterisch weniger anspruchsvolles Elektrophil schließen lassen könnte ${ }^{8)}$ - haben wir kein metaAcylierungsprodukt nachweisen können; dies spricht für ein sehr selektives Acylierungsagens. Im Gegensatz dazu werden sowohl unter Friedel-Crafts-Bedingungen ${ }^{7)}$ als auch bei der Acylierung von Toluol mit Benzoesäure-trifluormethansulfonsäure-anhydrid ${ }^{4)} 1-1.5 \%$ meta-Produkt gefunden.

Chem. Ber. 114 (1981) 


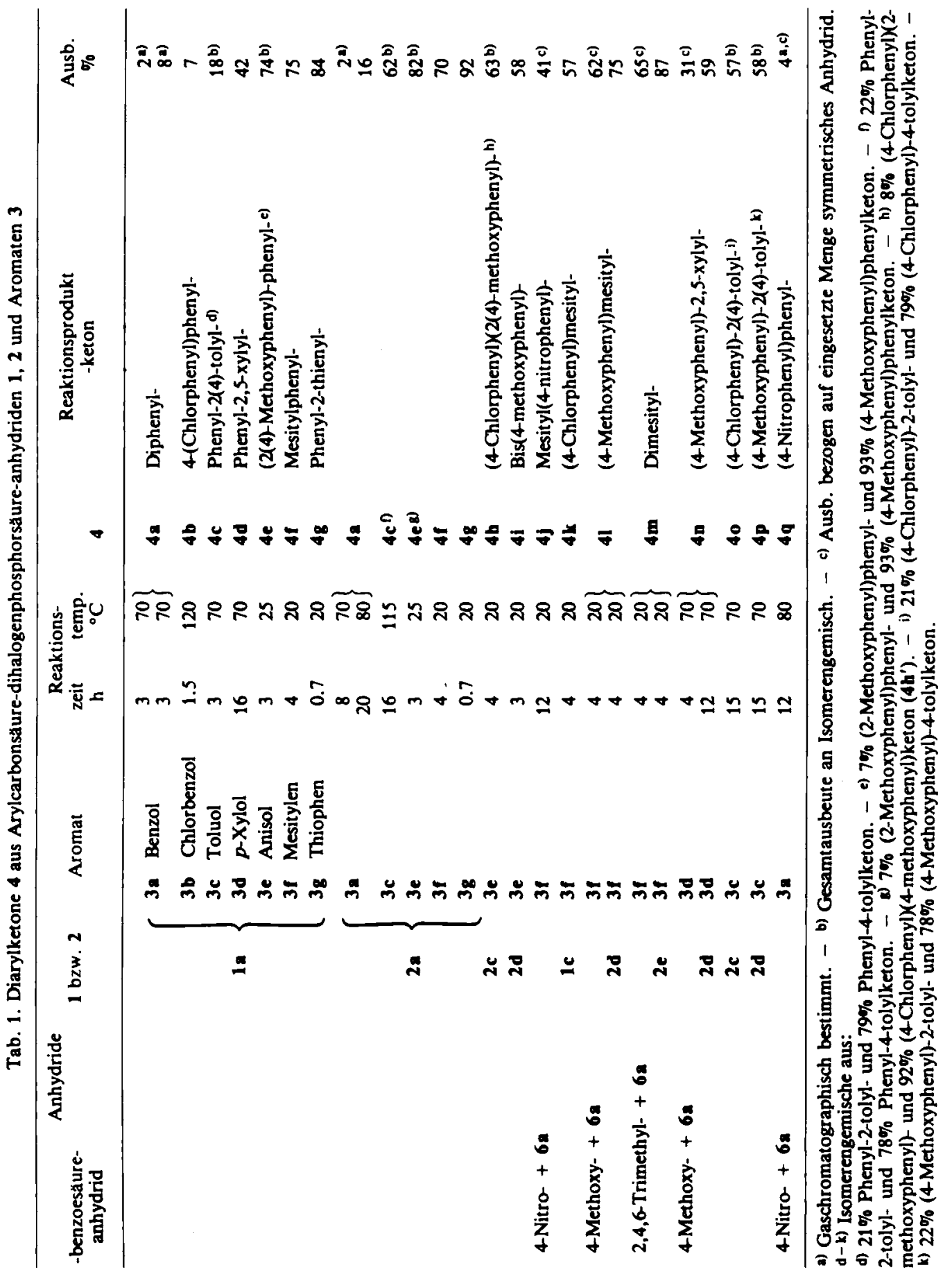

Chem. Ber. 114 (1981) 


\section{Acylierung mit Alkylcarbonsäure-dihalogenphosphorsäure-anhydriden} 7 bzw. 8

Die Reindarstellung der gemischten Anhydride $\mathbf{7}$ und $\mathbf{8}$ ist infolge ihrer Instabilität nicht gelungen ${ }^{3)}$. Wir haben daher die Acylierung von Aromaten mit den in situ hergestellten Anhydriden 7 und 8 einmal in der Weise durchgeführt, daß Gleichgewichtsgemische aus symmetrischen Anhydriden 5 und 6 und gemischten Anhydriden 7, 83) mit überschüssigen Aromaten umgesetzt wurden (Methode A) und zum anderen, indem symmetrische Anhydride 5 und 6 und der zu acylierende Aromat gemeinsam vorgelegt und bei Raumtemperatur umgesetzt wurden (Methode B) (Tab. 2).

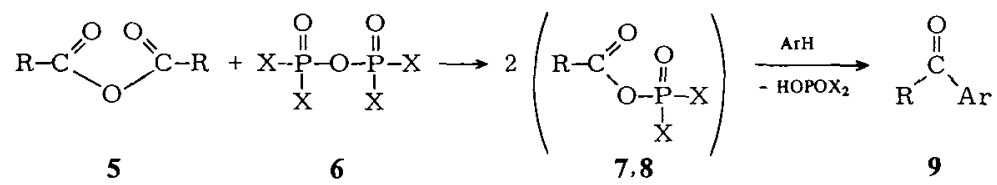

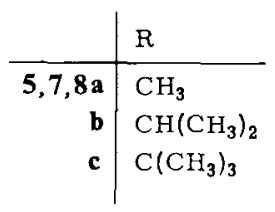

6a, 7: $\mathrm{X}=\mathrm{Cl}$ 6b, $8: X=F$

\begin{tabular}{r|ll} 
& $\mathrm{R}$ & $\mathrm{Ar}$ \\
\hline $\mathbf{9 a}$ & $\mathrm{CH}_{3}$ & $2,4,6-\left(\mathrm{CH}_{3}\right)_{3} \mathrm{C}_{6} \mathrm{H}_{2}$ \\
b & $\mathrm{CH}_{3}$ & $4-\mathrm{CH}_{3} \mathrm{OC}_{6} \mathrm{H}_{4}$ \\
c & $\mathrm{CH}_{3}$ & $2-\mathrm{C}_{4} \mathrm{H}_{3} \mathrm{~S}$ \\
d & $\mathrm{CH}\left(\mathrm{CH}_{3}\right)_{2}$ & $4-\mathrm{CH}_{3} \mathrm{OC}_{6} \mathrm{H}_{4}$ \\
e & $\mathrm{C}\left(\mathrm{CH}_{3}\right)_{3}$ & $2,4,6-\left(\mathrm{CH}_{3}\right)_{3} \mathrm{C}_{6} \mathrm{H}_{2}$ \\
f & $\mathrm{C}\left(\mathrm{CH}_{3}\right)_{3}$ & $4-\mathrm{CH}_{3} \mathrm{OC}_{6} \mathrm{H}_{4}$
\end{tabular}

Tab. 2. Alkylarylketone 9 aus Alkylcarbonsäure-dihalogenphosphorsäure-anhydriden 7a, 8a bzw. symmetrischen Alkylcarbonsäure- 5 und Dihalogenphosphorsäure-anhydriden 6 und Aromaten 3 bei Raumtemperatur

\begin{tabular}{|c|c|c|c|c|c|c|}
\hline $\begin{array}{l}\text { eingesetzte } \\
\text { Anhydride }\end{array}$ & Aromat & Methode & $\begin{array}{l}\text { Reakt.- } \\
\text { zeit h }\end{array}$ & & $\begin{array}{c}\text { Reaktionsprodukt } \\
\text {-keton }\end{array}$ & $\underset{\%}{\text { Ausb. }}$ \\
\hline $5 a+6 a+7 a$ & & A & $3)$ & & & 52 \\
\hline $5 a+6 b+8 a$ & 3f & A & $3\}$ & $9 \mathbf{a}$ & Mesitylmethyl- & 60 \\
\hline $5 a+6 a$ & & B & 3 & & & 60 \\
\hline $5 \mathbf{a}+6 \mathbf{a}$ & $\begin{array}{l}3 \mathbf{e} \\
\mathbf{3 g}\end{array}$ & $\begin{array}{l}\text { B } \\
\text { B }\end{array}$ & $\begin{array}{l}3 \\
3\end{array}$ & $\begin{array}{l}9 b \\
9 c\end{array}$ & $\begin{array}{l}\text { (4-Methoxyphenyl)methyl- } \\
\text { 4-Methyl-2-thienyl- }\end{array}$ & $\begin{array}{l}31 \\
43\end{array}$ \\
\hline $5 b+6 a$ & $3 e$ & B & 4 & 9d & Isopropyl(4-methoxyphenyl)- & 83 \\
\hline $5 c+6 a$ & $\begin{array}{l}3 \mathbf{f} \\
\mathbf{3 e}\end{array}$ & $\begin{array}{l}\text { B } \\
\text { B }\end{array}$ & $\begin{array}{l}8 \\
8\end{array}$ & $\begin{array}{l}9 \mathrm{e} \\
9 \mathrm{f}\end{array}$ & $\begin{array}{l}\text { tert-Butylmesityl- } \\
\text { tert-Butyl(4-methoxyphenyl)- }\end{array}$ & $\begin{array}{l}64 \\
78\end{array}$ \\
\hline
\end{tabular}

Aus den in Tab. 2 ersichtlichen Ausbeuten geht hervor, daß mit der wegen ihrer einfacheren Durchführbarkeit vorzuziehenden Methode B vergleichbar hohe Ausbeuten erreicht werden wie mit Methode A. Die im Vergleich zu den Verbindungen 1 und 2 noch größere Thermolabilität der Anhydride 7, 8 erfordert zur Vermeidung von Zersetzungsreaktionen noch niedrigere Umsetzungstemperaturen $\left(<25^{\circ} \mathrm{C}\right)$, so daß die weniger reaktiven Aromaten Benzol und Toluol mit 7 bzw. 8 nicht mehr acylierbar sind. Da bei den Acylierungsreaktionen mit den gemischten Anhydriden Dihalogenphosphor-

Chem. Ber. 114 (1981) 
săure freigesetzt wird, wăre es denkbar, daß insbesondere bei Anwendung der Methode B nicht die gemischten Anhydride 7, 8 reagieren, sondern daß die eingesetzten Carbonsäureanhydride 5 in Gegenwart der Dihalogenphosphorsăure die eigentlichen Acylierungsmittel sind. Um diesen eventuellen Anteil zu ermitteln, haben wir Mesitylen mit Acetanhydrid in Gegenwart von $15 \mathrm{~mol}-\%$ Difluorophosphorsäure bei $20^{\circ} \mathrm{C}$ umgesetzt .

Nach 3 Stunden waren jedoch nur Spuren von Mesitylmethylketon (9a) entstanden, so daß man annehmen muß, daß auch bei den nach Methode $B$ durchgeführten Reaktionen die gemischten Anhydride 7, 8 die reagierenden Acylierungsagentien sind.

\section{Acylierung mit Carbonsäuren und Dichlorophosphorsäure-anhydrid (6a)}

In der Literatur sind neben der ublichen Aromatenacylierung nach Friedel-Crafts mit Carbonsăurechloriden oder -anhydriden und molekularen Mengen Aluminiumchlorid auch Acylierungsreaktionen mit den Carbonsăuren selbst in Gegenwart von uberschüssigem Aluminiumchlorid beschrieben, wobei jedoch die primäre Bildung des Saurechlorids und anschließende Friedel-CraftsAcylierung angenommen wird 9). Reaktive Aromaten wie z. B. Phenol, Anisol, Alkylbenzole, aber auch Benzol selbst, lassen sich mit Carbonsăuren unter Zugabe von Bortrifluorid ${ }^{10 a)}$, Fluorwasserstoff ${ }^{10 b)}$ oder uberschüssiger Polyphosphorsăure ${ }^{10 c)}$ acylieren; Anisol bildet mit Gemischen aus Benzoyltrifluoracetat und Trifluoressigsăure bereits bei $60^{\circ} \mathrm{C}$ mit $64 \%$ Ausbeute $\left.\$ 1\right)$, mit Chloressigsaure und Acetanhydrid bei $170-180^{\circ} \mathrm{C}$ mit $88-90 \%$ Ausbeute ${ }^{12)}$ und mit Trichloressigsăure und Acetanhydrid bei $100^{\circ} \mathrm{C}$ mit $52 \%$ Ausbeute ${ }^{12)}$ (4-Methoxyphenyl)phenylketon; Thiophen ergibt mit Acetyltrifluoracetat bereits bei $45^{\circ} \mathrm{C}$ mit $88 \%$ Ausbeute Phenyl2-thienylketon 11 .

Im Rahmen unserer Untersuchungen zur Reindarstellung der gemischten Anhydride 1, 2 und $7,8^{3)}$ haben wir auch die Moglichkeit ihrer Bildung aus Carbonsäuren (Ameisen-, Essig-, Benzoe-, 2,4,6-Trimethylbenzoe- und Trifluoressigsăure) mit den Dihalogenphosphorsäure-anhydriden 6 geprüft. Dabei gelang es nur im Falle der starken Trifluoressigsäure, gemischte Dichloro- und Difluorophosphorsäure-trifluoressigsäure-anhydride mit ca. $10 \%$ Ausbeute ${ }^{31} \mathrm{P}$-NMR-spektroskopisch nachzuweisen.

Im Hinblick auf die oben angefuhrten Literaturhinweise haben wir trotz dieser Ergebnisse die Acylierung von Aromaten mit Gemischen aus Carbonsäuren und Dihalogenphosphorsäure-anhydriden untersucht. Wir setzten die Carbonsäuren mit der äquimolaren Menge Dichlorophosphorsäure-anhydrid (6a) und einem Úberschuß an Aromat um und konnten tatsächlich die entsprechenden Ketone 4 bzw. 9 isolieren (Tab. 3). Obwohl wir in den Reaktionsgemischen die gemischten Anhydride 1 bzw. 7 nicht nachgewiesen haben, nehmen wir sie auch hier als die eigentlich reagierenden Elektrophile an.

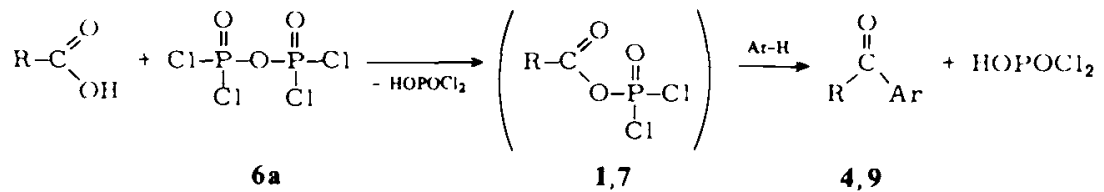

Die Umsetzungen der Benzoe- und 4-Nitrobenzoesäure mit dem reaktiven Mesitylen (3f) ergeben unter milden Reaktionsbedingungen $\left(20-40^{\circ} \mathrm{C}\right)$ Keton-Ausbeuten von über $80 \%$. Die Ausbeuten liegen damit um $40 \%$ höher als bei der Umsetzung von $3 \mathrm{f}$ mit einem Gemisch aus 4 Nitrobenzoesäure-anhydrid und $6 \mathrm{a}$ bei $20^{\circ} \mathrm{C}$ (s. Tab. 1). Der

Chem. Ber. //4(1981) 
Grund dafür könnte die schon bei der Bildung der gemischten Anhydride 1 in molaren Mengen entstehende Dichlorophosphorsäure sein, die durch Protonierung der Anhydride 1 zu reaktiveren Elektrophilen führt.

Tab. 3. Diarylketone 4 und Mesitylmethylketon (9a) aus Carbonsäuren, Dichlorophosphorsäureanhydrid (6a) und Aromaten 3

\begin{tabular}{|c|c|c|c|c|c|}
\hline $\begin{array}{c}\text { Carbonsäure } \\
\mathrm{R}\end{array}$ & Aromat & $\begin{array}{c}\mathrm{Re} \\
\text { zeit } \\
\mathrm{h}\end{array}$ & $\begin{array}{l}\text { ions- } \\
\text { temp. } \\
{ }^{\circ} \mathrm{C}\end{array}$ & $\begin{array}{c}\text { Reaktions- } \\
\text { produkt }\end{array}$ & $\underset{\%}{\text { Ausb. }}$ \\
\hline \multirow{4}{*}{$\mathrm{C}_{6} \mathrm{H}_{5}$} & $\mathbf{3 a}$ & 4 & 80 & $4 a$ & 18 \\
\hline & $3 f$ & 5 & 20 & $4 f$ & 85 \\
\hline & $3 g^{a)}$ & 20 & 12 & $4 g$ & 75 \\
\hline & $\mathbf{3 a}$ & 8 & 80 & $4 q$ & 7 \\
\hline \multirow[t]{2}{*}{$4 \mathrm{NO}_{2} \mathrm{C}_{6} \mathrm{H}_{4}$} & $3 f$ & 12 & $20\}$ & \multirow{2}{*}{$4 \mathbf{j}$} & 35 \\
\hline & $3 f$ & 8 & $40\}$ & & 86 \\
\hline $4-\mathrm{ClC}_{6} \mathrm{H}_{4}$ & $3 \mathbf{a}$ & 8 & 80 & $4 b$ & 4 \\
\hline $\mathrm{CH}_{3}$ & $\mathbf{3 f}$ & 3 & $40^{b)}$ & $9 a$ & 52 \\
\hline
\end{tabular}

a) In Methylenchlorid. - b) Reaktionswärme.

Wir danken dem Fonds der Chemischen Industrie für die Unterstützung dieser Arbeit.

\section{Experimenteller Teil}

Gaschromatographie: 5700 der Fa. Hewlett-Packard mit Flammenionisationsdetektor (FID), Integrator: Varian Aerograph 477 und Spectra-Physics Minigrator. Trägergas $30 \mathrm{ml} \mathrm{N} / \mathrm{min}$, Glassäule $2.30 \mathrm{~m}$ lang, $\varnothing 2 \mathrm{~mm}$. Stationäre Phase OV 101/5\% auf Gaschrom Q. Bei der gaschromatographischen Ausbeutebestimmung diente $m$-Dinitrobenzol als Standard.

${ }^{31}$ P-NMR-Spektren: Gerät WP $60(24.3 \mathrm{MHz})$ und WP $80(32.3 \mathrm{MHz})$ der Firma Bruker, $\delta$ [ppm] bezogen auf 85 proz. Phosphorsäure als externem Standard.

Diarylketone 4 (zu Tab. 1)

1) Aus Benzoesäure-dichloro- (1a) bzw. -difluorophosphorsäure-anhydrid (2a) und Aromaten 3: Unter Feuchtigkeitsausschluß $\left(\mathrm{P}_{4} \mathrm{O}_{10}\right)$ erwärmt man 1a bzw. 2a mit überschüssigem Aromat 3, zum Teil in Gegenwart von Nitromethan oder Methylenchlorid. Anschließend wird das Reaktionsgemisch mit ca. $50 \mathrm{ml} 2 \mathrm{~N} \mathrm{NaOH} 2 \mathrm{~h}$ bei Raumtemp. gerührt, mit $20-25 \mathrm{ml}$ Methylenchlorid ausgeschüttelt und die organische Phase mit $10 \mathrm{ml}$ Wasser gewaschen.

Aufarbeitung C: Nach Trocknen des Methylenchlorid-Extraktes über Magnesiumsulfat wird eingeengt und der Rückstand gaschromatographisch auf Reinheit und gegebenenfalls auf Isomerenverteilung untersucht und mit authentischen Produkten verglichen. Die weitere Reinigung erfolgt durch Destillation oder Umkristallisation.

Aufarbeitung $D$ : Der nicht getrocknete Methylenchlorid-Extrakt wird i. Vak. eingeengt, der Rückstand mit ca. $25 \mathrm{ml} 6 \mathrm{~N} \mathrm{KOH} 5 \mathrm{~h}$ auf $60-70^{\circ} \mathrm{C}$ unter Rühren erwärmt, anschließend mit ca. $100 \mathrm{ml}$ Wasser verdünnt, mit $30-50 \mathrm{ml}$ Methylenchlorid extrahiert und der Extrakt wie unter $C$ aufgearbeitet.

Chem. Ber. 114 (1981) 


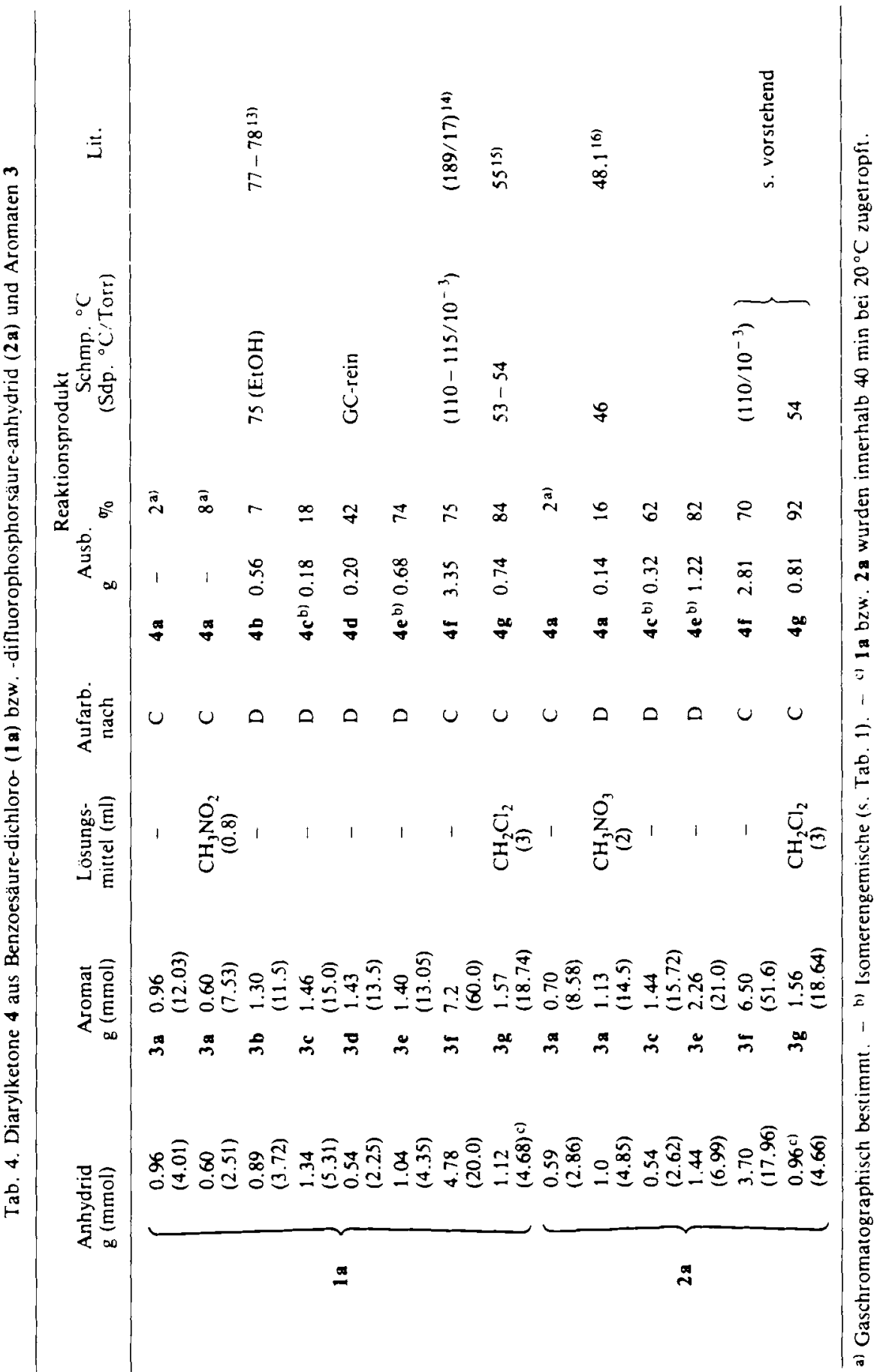

Chem. Ber. $/ 14$ (1981) 


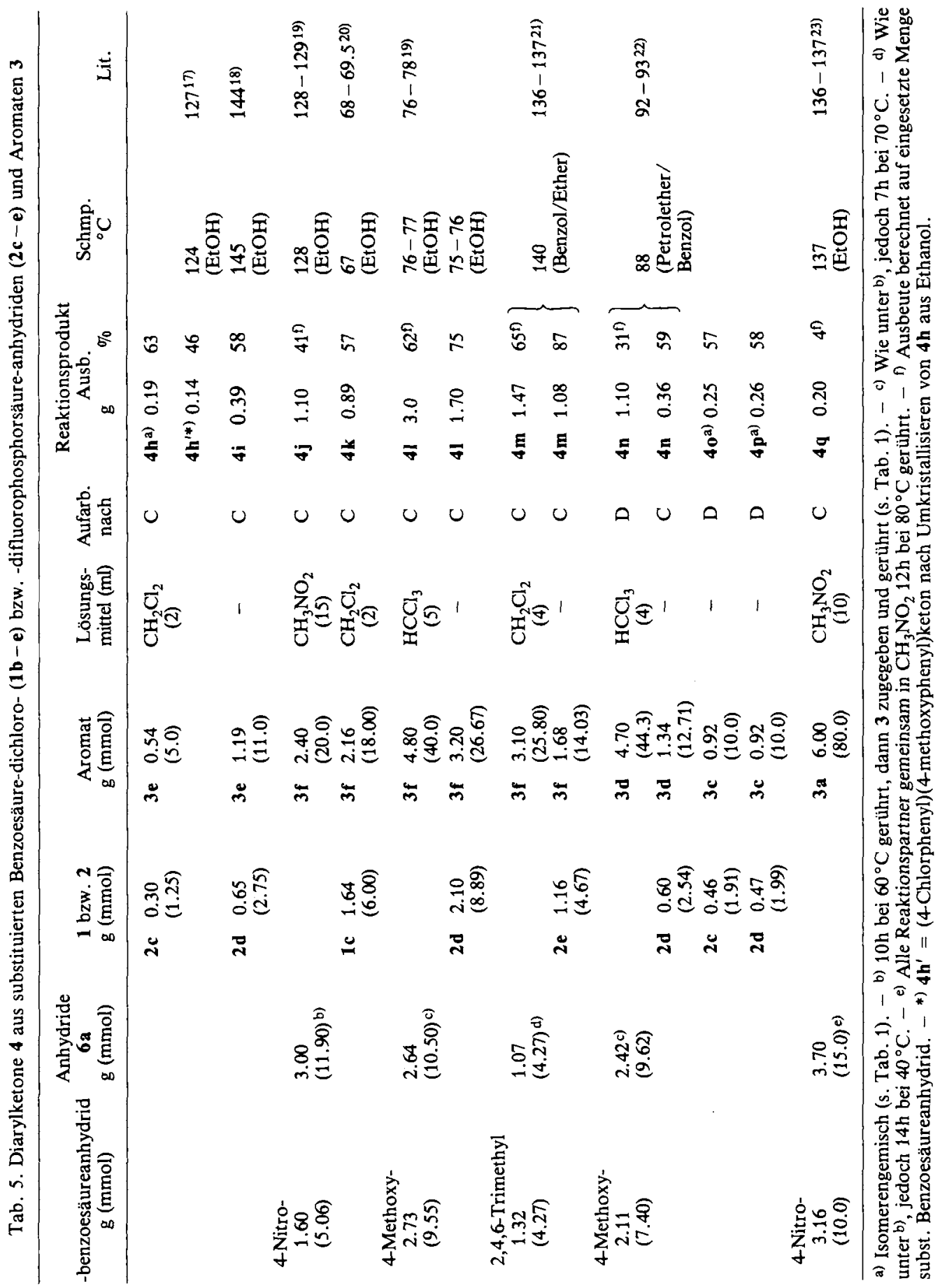

Chem. Ber. 114 (1981) 
2) Aus substituierten Benzoesaure-dichloro- $(\mathbf{1} \mathbf{b}-\mathbf{e})$ bzw. -difluorophosphorsaureanhydriden (2c-e) und Aromaten 3: Wie vorstehend beschrieben, jedoch werden anstelle von 1 oder 2 zum. Teil die symmetrischen Anhydride 4-Nitrobenzoesäure-, 4-Methoxybenzoesäure- bzw. 2,4,6Trimethylbenzoesáure-anhydrid und Dichlorophosphorsaure-anhydrid (6a) vorgelegt und unter den angegebenen Bedingungen (s. Tab. 1) gerührt. Anschließend gibı man 3 zu und arbeitet wic vorstehend beschricben weiter.

\section{Alkylarvlketone 9 (zu Tab. 2)}

Methode A: Man läßt aquimolare Mengen Acetanhydrid (5a) und Dichloro- (6a) bzw. Difluorophosphorsäure-anhydrid (6b) $3 \mathrm{~h}$ bei Raumtemp. stehen (Trockenrohr, $\mathrm{P}_{4} \mathrm{O}_{10}$ ), troptl das erhaltene Reaktionsgemisch innerhalb $10 \mathrm{~min}$ zu überschüsigem Mesitylen (3f), gießt nach $3 \mathrm{~h}$ Stehenlassen bei Raumtemp. in $100 \mathrm{ml} 2 \mathrm{~N} \mathrm{NaOH}$, rührt $1 \mathrm{~h}$ bei Raumtemp., schüttelt mit $50 \mathrm{ml}$ Ether aus, trocknet die organische Phase über Magnesiumsulfat und destilliert.

Methode B: Wie vorstehend beschrieben, jedoch laßt man das Reaktionsgemisch aus 5, 6a und 3 bei Raumtemp. die angegebene Zeit stehen. Die Aufarbeitung erfolgt analog Methode A.

Tab. 6. Alkylarylketone 9 aus symmetrischen Alkylcarbonsäure- (5a-c) und Dihalogenphosphorsaure-anhydriden 6 und Aromaten 3

\begin{tabular}{|c|c|c|c|c|c|c|c|c|c|c|}
\hline & $\begin{array}{l}\text { Anh } \\
(\mathrm{g} / \mathrm{r}\end{array}$ & $\begin{array}{l}\text { dride } \\
\text { mol) }\end{array}$ & & Aromat & Metho- & & Aus & & $\begin{array}{l}\text { Reaktionsprod } \\
\text { Sdp. }{ }^{\circ} \mathrm{C} / \text { Torr }\end{array}$ & ukt \\
\hline & 5 & $6 a$ & & $(\mathrm{mmol})$ & & & $\mathrm{g}$ & $\%$ & (Schmp.) & \\
\hline $5 \mathbf{a}$ & $\begin{array}{l}1.02 \\
(10.0)\end{array}$ & $\begin{array}{l}2.52 \\
(10.0)\end{array}$ & $3 f^{a)}$ & $\begin{array}{l}3.60 \\
(30.0)\end{array}$ & A & $9 \mathbf{a}$ & 1.70 & 52 & & \\
\hline $5 \mathbf{a}$ & $\begin{array}{l}0.29 \\
(2.84)\end{array}$ & $\begin{array}{l}0.52^{h)} \\
(2.84)\end{array}$ & $3 f$ & 0.92 & A & $9 \mathbf{a}$ & 0.55 & 60 & $100-105 / 10$ & $120 / 12^{241}$ \\
\hline $5 \mathbf{a}$ & $\begin{array}{l}2.04 \\
(20.0)\end{array}$ & $\begin{array}{l}5.04 \\
(20.0)\end{array}$ & $3 \mathbf{f}$ & $\begin{array}{l}5.80 \\
(48.33)\end{array}$ & B & $9 \mathbf{a}$ & 4.0 & 60 & & \\
\hline $5 \mathbf{a}$ & $\begin{array}{l}1.02 \\
(10.0)\end{array}$ & $\begin{array}{l}2.52 \\
(10.0)\end{array}$ & $3 \mathbf{e}$ & $\begin{array}{l}5.40 \\
(50.0)\end{array}$ & $\mathrm{B}$ & $9 \mathbf{b}$ & 0.93 & 31 & $126-128 / 12$ & $138-139^{241}$ \\
\hline $5 \mathbf{a}$ & $\begin{array}{l}1.02 \\
(10.0)\end{array}$ & $\begin{array}{l}2.52 \\
(10.0)\end{array}$ & $3 g^{()}$ & $\begin{array}{l}2.52 \\
(30.0)\end{array}$ & $\mathrm{B}$ & $9 \mathrm{c}$ & 1.15 & 43 & $86-88 / 10$ & $89 / 9^{15}$ \\
\hline $5 b$ & $\begin{array}{l}3.16 \\
(20.0)\end{array}$ & $\begin{array}{l}5.04 \\
(20.0)\end{array}$ & $3 e$ & $\begin{array}{l}7.56 \\
(70.0)\end{array}$ & B & $9 d$ & 5.9 & 83 & $135-136 / 12$ & $152.5-153 / 16^{25}$ \\
\hline $5 c$ & $\begin{array}{l}3.72 \\
(20.0)\end{array}$ & $\begin{array}{l}5.04 \\
(20.0)\end{array}$ & $3 f$ & $\begin{array}{l}7.20 \\
(60.0)\end{array}$ & B & $9 e$ & 5.3 & 64 & $130-132 / 10$ & $120-122 / 8^{26)}$ \\
\hline $5 \mathrm{c}$ & $\begin{array}{l}1.86 \\
(10.0)\end{array}$ & $\begin{array}{l}2.52 \\
(10.0)\end{array}$ & $3 e$ & $\begin{array}{l}5.40 \\
(50.0)\end{array}$ & $\mathrm{B}$ & $9 f$ & 3.0 & 78 & $139 / 11$ & $138-140 / 8^{27)}$ \\
\hline
\end{tabular}

a) In $10 \mathrm{ml}$ Methylenchlorid. - h) Difluorophosphorsaure-anhydrid (6b) in $3 \mathrm{ml}$ Methylenchlorid $1 \mathrm{~h}$ bei $0^{\circ} \mathrm{C}$. - C In $5 \mathrm{~m}$ ! Benzol innerhalb $2 \mathrm{~h}$ zugetropft.

Umsetzung von Acetanhydrid (5a) mit Mesitylen (3f) in Gegenwart ion Diflworophosphorsăure: Man läßt zu $3.60 \mathrm{~g}$ (30.0 mmol) $3 \mathrm{f}$ und $0.15 \mathrm{~g}(1.47 \mathrm{mmol})$ Difluorophosphorsäure bei

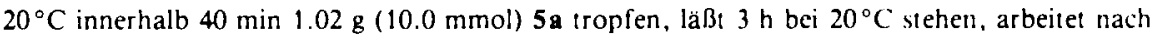
Methode $\mathrm{A}$ auf und untersucht das Reaktionsgemisch gaschromatographisch.

Umsetzung ion Trifluoressigsaure mit Dichloro- (6a) bzw. Difluorophosphorsäure-anhydrid (6b): Man lảßı $5.04 \mathrm{~g}(20.0 \mathrm{mmol})$ Trifluoressigsăure und $2.28 \mathrm{~g}(20.0 \mathrm{mmol}) 6 \mathrm{a} \mathrm{bzw.} 0.90 \mathrm{~g}$ $(5.0 \mathrm{mmol})$ Trifluoressigsäure und $0.55 \mathrm{~g}(5.0 \mathrm{mmol}) 6 \mathrm{~b}$ jeweils $4 \mathrm{~h}$ bei $20^{\circ} \mathrm{C}$ stehen und untersucht anschließend die Reaktionsgemische ${ }^{31}$ P-NMR-spektroskopisch. Man findet jeweils 10\% Trifluoressigsäure-dichloro- $(\delta=2.8) \mathrm{bzw}$. -difluorophosphorsäure-anhydrid $\left(\delta=33.2, J_{\mathrm{PY}}=\right.$ $1080 \mathrm{~Hz})$. 
Tab. 7. Diarylketone 4 und Mesitylmethylketon (9a) aus Carbonsäuren, Dichlorophosphorsäure-anhydrid (6a) und Aromaten 3

\begin{tabular}{|c|c|c|c|c|c|c|c|c|}
\hline \multirow{3}{*}{-säure } & \multirow{3}{*}{$\begin{array}{l}\mathrm{g}(\mathrm{mmol}) \\
1.22 \\
(10.0)\end{array}$} & \multirow{3}{*}{$\begin{array}{l}\begin{array}{c}6 a \\
\mathrm{~g}(\mathrm{mmol})\end{array} \\
\begin{array}{l}2.52 \\
(10.0)\end{array}\end{array}$} & \multirow{2}{*}{\multicolumn{2}{|c|}{$\begin{array}{c}3 \\
\mathrm{~g}(\mathrm{mmol})\end{array}$}} & \multicolumn{3}{|c|}{ Reaktionsprodukt } & \multirow[b]{2}{*}{ Lit. } \\
\hline & & & & & & $\begin{array}{l}\text { dusb. } \\
\mathrm{g}\end{array}$ & $\underset{{ }^{\circ} \mathrm{C}}{\text { Schmp. }}$ & \\
\hline & & & $3 \mathbf{a}$ & $\begin{array}{l}2.34 \\
(30.0)\end{array}$ & $4 a$ & 0.32 & 46 & $48^{16)}$ \\
\hline Benzoe- & $\begin{array}{l}2.44 \\
(20.0)\end{array}$ & $\begin{array}{l}5.04 \\
(20.0)\end{array}$ & $3 f$ & $\begin{array}{l}12.0 \\
(100.0)\end{array}$ & $4 f$ & 3.80 & s. Tab. 4 & \\
\hline Benzoe- & $\begin{array}{l}1.22 \\
(10.0)\end{array}$ & $\begin{array}{l}2.52 \\
(10.0)\end{array}$ & $3 \mathbf{g}$ & $\begin{array}{l}1.68 \\
(20.0)^{a)}\end{array}$ & $4 \mathrm{~g}$ & 1.42 & & \\
\hline $\begin{array}{l}\text { 4-Nitro- } \\
\text { benzoe- }\end{array}$ & $\begin{array}{l}3.90 \\
(23.3)\end{array}$ & $\begin{array}{l}5.80 \\
(23.3)\end{array}$ & $\mathbf{3 a}$ & $\begin{array}{l}12.0 \\
(100.0)\end{array}$ & $4 q$ & 0.38 & $137-138$ & $138^{23)}$ \\
\hline $\begin{array}{l}\text { 4-Nitro- } \\
\text { benzoe- }\end{array}$ & $\begin{array}{l}3.34 \\
(20.0)\end{array}$ & $\begin{array}{l}5.04 \\
(20.0)\end{array}$ & $3 \mathbf{f}$ & $\begin{array}{l}12.0 \\
(100.0)\end{array}$ & $4 j$ & $\begin{array}{l}1.88^{b)} \\
\left.4.65^{c}\right)\end{array}$ & s. Tab. 5 & \\
\hline $\begin{array}{l}\text { 4-Chlor- } \\
\text { benzoe- }\end{array}$ & $\begin{array}{l}1.55 \\
(10.0)\end{array}$ & $\begin{array}{l}2.52 \\
(10.0)\end{array}$ & $\mathbf{3 a}$ & $\begin{array}{l}6.24 \\
(80.0)\end{array}$ & $4 b$ & 0.08 & $.1 \mathrm{ab} .4$ & \\
\hline Essig- & $\begin{array}{l}1.20 \\
(20.0)\end{array}$ & $\begin{array}{l}5.04 \\
(20.0)\end{array}$ & 3f & $\begin{array}{l}3.60 \\
(30.0)\end{array}$ & $9 \mathbf{a}$ & 1.75 & s. Tab. 6 & \\
\hline
\end{tabular}

a) In $5 \mathrm{ml}$ Methylenchlorid. - b) $12 \mathrm{~h}$ bei $20^{\circ} \mathrm{C}$. - c) $8 \mathrm{~h}$ bei $40^{\circ} \mathrm{C}$.

Umsetzungen von Carbonsäuren mit Dichlorophosphorsäureanhydrid (6a) und Aromaten 3 (zu Tab. 3): Äquimolare Mengen Carbonsäure und 6a werden mit einem Überschuß an 3 bei der angegebenen Temp. (s. Tab. 3) gerührt ( $\mathrm{P}_{4} \mathrm{O}_{10}$-Trockenrohr). Nach Einrühren des Reaktionsgemisches in $100-150 \mathrm{ml} 2 \mathrm{~N} \mathrm{NaOH}$ und $3 \mathrm{~h}$ Rühren wird die organische Phase in $20 \mathrm{ml}$ Methylenchlorid aufgenommen, die Lösung mit $10 \mathrm{ml}$ Wasser gewaschen und über Magnesiumsulfat getrocknet. Nach Abdestillieren des Lösungsmittels wird i. Vak. destilliert und umkristallisiert.

1) 19. Mitteil.: Zur elektrophilen Aromatensubstitution; 18. Mitteil.: $F$. Effenberger, $U . K u \beta-$ maul und K. Huthmacher, Chem. Ber. 112, 1677 (1979).

2) G. König, Teil der Dissertation, Univ. Stuttgart 1978.

3) F. Effenberger und $G$. Konnig, Chem. Ber. 114, 916 (1981), vorstehend.

4) 4a) F. Effenberger und G. Epple, Angew. Chem. 84, 294 (1972); Angew. Chem., Int. Ed. Engl. 11, 299 (1972). - 4b) G. Epple, Dissertation, Univ. Stuttgart 1972.

5) E. Fluck und E. Beuerle, Z. Anorg. Allg. Chem. 411, 125 (1975).

6) F. Effenberger, G. König und H. Klenk, Angew. Chem. 90, 740 (1978); Angew. Chem., Int. Ed. Engl. 17, 695 (1978).

7) H. C. Brown und F. R. Jensen, J. Am. Chem. Soc. 80, 2296 (1958).

8) F. Effenberger, Angew. Chem. 92, 147 (1980); Angew. Chem., Int. Ed. Engl. 19, 151 (1980).

9) P. H. Groggins, R. H. Nagel und A. J. Stirton, Ind. Eng. Chem. 26, 1317 (1934).

10) 10a) V. Gold und T. Riley, J. Chem. Soc. 1961, 1676. - 10b) J. H. Simons, D. I. Randall und S. Archer, J. Am. Chem. Soc. 61, 1795 (1939). - 10c) P. D. Gardner, ebenda 76, 4550 (1954).

11) E. J. Bourne, M. Stacey, J. C. Tatlow und R. Worrall, J. Chem. Soc. 1954, 2011.

12) F. Unger, Liebigs Ann. Chem. 504, 267 (1933).

13) R. C. Weast, Handbook of Chemistry and Physics, C-201, 57. Ed. CRC-Press, Cleveland Ohio 1977.

14) A. Klages und P. Allendorf, Ber. Dtsch. Chem. Ges. 31, 1001 (1889).

15) G. Stadnikow und I. Goldfarb, Ber. Dtsch. Chem. Ges. 61, 2341 (1928).

16) Lit. ${ }^{13)}, \mathrm{C}-200$.

17) P. P. Peterson, Am. Chem. J. 46, 339 (1911), Chem. Abstr. 5, 3800 (1911). 
18) $H$. Schnackenberg und $R$. Scholl, Ber. Disch. Chem. Ges. 36, 654 (1903).

19) O. Korver, J. U. Veeland und Th. I. de Boer, Rec. Trav. Chim. Pays-Bas 84, 289 (1965).

20) $R$. C. Fuson und M. D. Armstrong, J. Am. Chem. Soc. 63, 2650 (1941).

21) E. P. Kohler und R. Baltzly, J. Am. Chem. Soc. 54, 4023 (1932).

22) $K$. M. Baker und B. R. Davis, Tetrahedron 25, 2491 (1969).

23) H. Adkins, R. M. Elofson, A. G. Rossow und C. C. Robinson, J. Am. Chem. Soc. 71, 3622 (1949).

24) Lit. ${ }^{13)}, \mathrm{C}-101$.

25) Lit. ${ }^{22)}$, S. 280.

26) D. V. Nightingale, R. L. Sublett, R. A. Carpenter und H. D. Radford, J. Org. Chem. 16, 655 (1951).

27) E. Rothstein und $R$. W. Saville, J. Chem. Soc. 1949, 1953.

[219/80] 